\title{
The role of testing in the preoperative evaluation
}

\section{ABSTRACT}

Preoperative laboratory and electrocardiographic testing should be driven by the patient's history and physical examination and the risk of the surgical procedure. A test is likely to be indicated only if it can correctly identify abnormalities and will change the diagnosis, the management plan, or the patient's outcome. Needless testing is expensive, may unnecessarily delay the operation, and puts the patient at risk for unnecessary interventions. Preoperative evaluation centers can help hospitals standardize and optimize preoperative testing while fostering more consistent regulatory documentation and appropriate coding for reimbursement.

\section{KEY POINTS}

Age-based criteria for preoperative testing are controversial because test abnormalities are common in older people but are not as predictive of complications as information gained from the history and physical exam.

Pregnancy testing is an example of an appropriate preoperative test because pregnancy is often not detectable by the history and physical exam and a positive result would affect case management.

Routine ordering of preoperative electrocardiograms is not recommended because they are unlikely to offer predictive value beyond the history and physical exam and are costly to an institution over time.

Routine and aged-based preoperative tests are no longer reimbursed by the Centers for Medicare and Medicaid Services.
D. outine presurgical assessment of patients with a standard battery of tests not only is wasteful but can lead to more unnecessary expense, delay, and even risk to the patient and physician. Any abnormal tests results, even if likely to be clinically unimportant to the upcoming surgery, will need to be followed up to rule out a significant abnormality that may have later implications. This review will outline strategies for making decisions about which tests are clinically useful for preoperative assessment of a given patient and also discuss the value of preoperative evaluation centers in promoting appropriate preoperative testing.

\section{PREOPERATIVE EVALUATION SHOULD BE CLEARLY DIRECTED}

Most patients scheduled for surgery at Brigham and Women's Hospital are assessed by the staff at our preoperative evaluation center. We take a medical history and conduct a physical examination, review the medical records, order laboratory tests or other studies as indicated, and determine which patients need further workup or consultations. The goals are to evaluate patient readiness for anesthesia and surgery, optimize patient health before surgery, enhance the quality of perioperative care, reduce the morbidity of surgery and length of stay, and return the patient to normal functioning. ${ }^{1,2}$

The above goals are generally achieved by directed laboratory testing, managing the patient's medications, stabilizing disorders when possible, and creating plans for postoperative care and pain management. Communication among the surgeon, the anesthesiology team, and the preoperative medical consultant (if there is one) is critical..$^{1,2}$

In contrast, "clearing the patient for surgery" is not a legitimate goal of consultation. The real issues to be taken up in a consult are:

- What is the patient's risk of complications (cardiac and noncardiac)?

- Would further risk stratification alter patient management?

- Can anything be done to reduce the patient's risk?

If indicated, a consult should cover the entire perioperative period, offering opinions on operative risk and suggesting treatments that affect long-term patient outcomes. Rarely is preoperative intervention necessary 
just to lower the risk of surgery. Most interventions that are needed should be done regardless of the surgery.

Everyone on the medical team should have the goal of efficient resource utilization, including avoidance of unnecessary visits, laboratory testing, and consultations.

\section{PREOPERATIVE TESTING: WHAT IS NEEDED?}

Preoperative testing is extremely expensive: even more than 20 years ago, preoperative medical testing for all types of surgery accounted for approximately $\$ 30$ billion in US health care costs annually. ${ }^{3}$ The likelihood of abnormal test results increases with age, and the more tests performed, the more likely a false positive will occur, further driving up costs.

Preoperative testing should generally be directed by a targeted history and physical examination, and the relevance of any tests should be considered in light of the type of procedure that is planned, particularly the hemodynamic changes and blood loss involved. Before ordering a test, physicians should be sure that there is a good reason for the test, that it is consistent with established guidelines, and that the results will be useful (ie, have the potential to change management).

Case study: Inguinal surgery in a healthy elderly man A 72-year-old man is being evaluated prior to a right inguinal herniorrhaphy. He has osteoarthritis but is otherwise healthy and jogs 3 to 5 miles several times a week. He takes no medications and has no known drug allergies.

Question: Which of the following tests is necessary prior to surgery?

A. Complete blood cell count (CBC)

B. Prothrombin time and partial thromboplastin time

C. Electrocardiogram (ECG)

D. All of the above

E. None of the above

The correct answer is $\mathrm{E}$ (none of the above), for the reasons laid out in the following section.

\section{Unnecessary testing may cause more harm than good} Untargeted testing should be avoided. An unexpected result will probably not be clinically significant for the surgery and will only lead to more needless testing, unnecessary anxiety for the patient, and delays in proceeding to the operating room. ${ }^{4}$ The more tests that are ordered, the higher the likelihood of having an abnormal result by chance: for a test with $95 \%$ specificity, results for 1 out of 13 ordered tests will likely be abnormal without there being a true underlying physiologic abnormality.

Researchers at Johns Hopkins University assessed the value of routine preoperative medical testing in a randomized study of nearly 20,000 patients undergoing elective cataract surgery whose preoperative history and physical examination was either preceded or not preceded by a standard battery of tests, including ECG, CBC, electrolytes, urea nitrogen, creatinine, and glucose. ${ }^{5}$ This was an ideal study population, given the relatively noninvasive nature of the procedure (with minimal hemodynamic changes) and cataract patients' relatively advanced age and resulting likelihood of comorbidities. Notably, there were no differences between the two groups in the overall rate of complications (approximately 3\%), which led the researchers to conclude that routine preoperative medical testing does not increase the safety of cataract procedures. These results could be applied to other low-risk cases.

Unnecessary testing is also expensive. Researchers at Stanford University Hospital retrospectively compared preoperative test orders during 6-month periods before and 1 year after development of an anesthesia preoperative evaluation clinic. ${ }^{6}$ They found a $55 \%$ reduction in the number of preoperative tests ordered from the period before the clinic was established, when tests were ordered by surgeons and primary care physicians, to the period after the clinic was established, when test ordering was transferred to anesthesiologists at the clinic. This reduction in the number of tests ordered resulted in a 59\% reduction in the hospital's expenditures for preoperative tests, yielding $\$ 112$ in cost savings per patient. No operating room cancellations, delays, or adverse patient events were reported as a result of the change.

Similar results were reported more recently by researchers at a Canadian hospital, who found that selective preoperative test ordering by staff anesthesiologists reduced the number and cost of preoperative studies compared with usual practice without a resulting increase in complications. ${ }^{?}$

\section{What are the real legal risks?}

Many surgeons express the fear that they will be sued if they do not routinely order preoperative tests. My view is that from a medicolegal standpoint, it is usually better not to order an unnecessary test if the next step to take in the event of an abnormal result would be unclear. The legal risk is greater for not following an abnormal test result than for not ordering a test that was not indicated. One may uncover an abnormal laboratory test finding that is not likely to be clinically significant but that could result in legal action if it were not evaluated further. A complication that may not be related to the abnormal result may develop at some point in the future and be blamed on the lack of follow-up. At our center, we insist that when a physician orders a test, he or she is responsible for the results and for following up on abnormalities.

\section{Should testing be based on age?}

Using age as a criterion for preoperative testing is controversial. There is no doubt that the older a patient is, the more likely he or she is to have abnormal test results: patients aged 70 years or older have about a 10\% 
TABLE 1

Criteria for determining whether a preoperative test is indicated*

Diagnostic efficacy: Does the test correctly identify abnormalities?

Diagnostic effectiveness: Would the test change your diagnosis?

Therapeutic efficacy: Would the test change your management?

Therapeutic effectiveness: Would the test change the patient's outcome?

*Adapted from Silverstein and Boland. ${ }^{11}$

chance of having abnormal levels of serum creatinine, hemoglobin, or glucose ${ }^{8}$ and a $75 \%$ chance of having at least one abnormality on their ECG (and a 50\% chance of having a major ECG abnormality). ${ }^{9}$ However, these factors were found not to be predictive of postoperative complications. In contrast, predictive factors for this age group are an American Society of Anesthesiologists (ASA) physical status classification of at least 3 (indicating severe systemic disease), the risk of the surgical procedure, and a history of congestive heart failure. ${ }^{8,9}$

\section{Guidelines for testing - and for not testing}

About 10 years ago, the ASA attempted to develop a practice guideline for routine preoperative testing. The available data were so inconsistent, however, that the ASA could not reach a consensus and instead issued a practice advisory. ${ }^{10}$

Even so, there are a number of general principles for avoiding unnecessary preoperative testing:

- Routine laboratory tests are not good screening devices and should not be used to screen for disease

- Repetition should be avoided: there is no need to repeat a recent test

- Healthy patients may not need testing

- Patients undergoing minimally invasive procedures may not need testing

- A test should be ordered only if its results will influence management.

Table 1 lists four criteria for making an educated decision about whether a preoperative test is indicated. ${ }^{11} \mathrm{In}$ general, a test that meets only one or none of the four criteria is probably not a good test, and if it meets three or four of the criteria, it is a very good test (meeting two criteria would be borderline). These criteria should always be considered when ordering a laboratory test, an ECG, a stress test, or an additional consult.

\section{A CLOSER LOOK AT A FEW SPECIFIC TESTS}

Question: Which of the following tests is most likely to provide useful information to aid clinical decision- making during a preoperative evaluation for laparoscopic cholecystectomy?

A. A chest radiograph in a 43-year-old woman with asthma B. An ECG in a 71-year-old man with hypertension C. A pregnancy test in an 18-year-old woman with amenorrhea D. A prothrombin time in a 51-year-old man with anemia E. A urinalysis in a 67-year-old woman with diabetes

The best answer is $\mathrm{C}$ (pregnancy test); an ECG in the 71-year-old man would be less useful (see below). The remaining choices - chest radiograph, prothrombin time, and urinalysis-are even less appropriate. A chest radiograph in an asthmatic patient is not likely to yield more information than what is obtained from the history and physical exam. Patients with anemia are not likely to have abnormal coagulation, and the role of urinalysis in detecting glucose and protein in asymptomatic diabetic patients is limited.

\section{Routine pregnancy testing is justifiable}

There are a number of reasons to justify a low threshold for preoperative pregnancy testing ${ }^{10}$ :

- Patients, especially adolescents, are often unreliable in suspecting that they might be pregnant (in several studies of routine preoperative pregnancy screening, $0.3 \%$ to $2.2 \%$ of tests were positive)

- History and physical examination are often insufficient to determine early pregnancy

- Management usually changes if it is discovered that a patient is pregnant.

Using the four criteria from Table 1, pregnancy testing rates high as a useful test: it would identify "abnormality," it would determine a diagnosis, and it would likely change management.

\section{Routine ECG has limited utility}

In contrast, routine preoperative ECG is not well supported. A recent study from the Netherlands assessed the added value of a preoperative ECG in predicting myocardial infarction and death following noncardiac surgery among 2,422 patients older than age 50 years. ${ }^{12}$ It showed that ECG findings were no more predictive of complications than findings from the history and physical examination and the patient's activity level.

From our own data at Brigham and Women's Hospital, ${ }^{13}$ we found that the presence of any of the following six risk factors predicted all but $0.44 \%$ of ECG abnormalities in patients aged 50 years or older presenting for preoperative evaluation:

- Age greater than 65 years

- Congestive heart failure

- High cholesterol

- Angina

- Myocardial infarction

- Severe valvular disease. 
The 2007 guidelines on perioperative risk assessment from the American College of Cardiology and American Heart Association (ACC/AHA) do not consider ECG to be indicated in asymptomatic patients undergoing low-risk noncardiac procedures regardless of patient age, ${ }^{14}$ like the 71 -year-old man with hypertension in the above case question. These guidelines also state that evidence for routine ECG orders is not well established in patients with at least one clinical risk factor undergoing intermediate-risk procedures.

The aforementioned ASA practice advisory acknowledges that the likelihood of ECG abnormalities rises with increasing patient age, but the ASA was unable to reach consensus on a minimum age for routinely ordering an ECG in surgical candidates. ${ }^{10}$ The advisory recommends taking into account other factors, such as cardiac risk factors, the presence of cardiocirculatory or respiratory disease, and the type and invasiveness of the surgical procedure. ${ }^{10}$

In recommendations not specific to the perioperative setting, the US Preventive Services Task Force advises against routine screening for coronary heart disease with ECG or exercise treadmill testing. ${ }^{15}$ It gives routine screening a "D" recommendation, indicating that risk is greater than benefit because of the potential for unnecessary invasive procedures, overtreatment, and mislabeling of patients.

Our group at Brigham and Women's Hospital recently surveyed anesthesiology program directors at US teaching hospitals to determine their preoperative test-ordering practices. ${ }^{16}$ Among the 75 respondents (58\% response rate), $95 \%$ said their institutions have no requirements for ordering ECGs unless indicated based on age, history, or surgery type; $71 \%$ said their institutions have age-based requirements for ordering ECGs (usually > 50 years). Most respondents reported that their institutions are ordering fewer ECGs since the publication of the 2007 ACC/AHA guidelines on perioperative evaluation.

Whether or not age should be used as a criterion for ECG testing is controversial, and editorials on this subject abound. ${ }^{17-19}$ They point out that clinicians must be careful before abandoning routine ECGs in elderly patients, for several reasons:

- An abnormal ECG (or abnormal lab test results) may modify a patient's ASA classification (which is predictive of complications)

- At least one-quarter of myocardial infarctions in elderly persons are "silent" or clinically unrecognized

- A preoperative ECG provides a useful baseline if the patient should develop ECG changes, chest pain, or cardiac complications during the perioperative period.

Most institutions use age as a criterion for ordering tests, especially for ECGs. If such a policy is used, a threshold of 60 years or older is probably most appropriate. However, a patient with good functional capacity who is undergoing a low-risk procedure does not need cardiac testing. ${ }^{14,20}$

An additional consideration is cost. Although the cost of a single ECG is modest, the cumulative cost of preoperative ECGs for all older surgical patients is significant over the course of a year. Because the Centers for Medicare and Medicaid Services (CMS) no longer cover routine preoperative ECGs, routine testing can be very costly to an institution over time.

\section{COST AND REGULATORY BENEFITS OF PREOPERATIVE CENTERS}

Preoperative evaluation centers tend to be cost-effective, as they keep consultations and redundant provider interviews to a minimum, encourage more appropriate targeting of tests, and help to avoid last-minute operating room delays and cancellations. ${ }^{21,22}$ They also provide an efficient means of compiling the chart for the operating room.

\section{The merits of standardization}

Preoperative evaluation centers likewise encourage more standardized preoperative assessment, which can facilitate compliance with surgical quality measures such as those from the National Surgical Quality Improvement Program and the Leapfrog Group. Standardization also fosters more efficient and consistent regulatory documentation, making it easier to follow requirements from CMS (often linked to reimbursement) and the Joint Commission. It also tends to improve reimbursement by encouraging more appropriate coding under CMS' diagnosis-related group (DRG) system to indicate that whatever testing is ordered is related to the surgical diagnosis or to relevant comorbidities.

No excessive dictates from Joint Commission or CMS Contrary to what many believe, the Joint Commission does not require excessive preoperative testing. The Joint Commission has no mandate for routine diagnostic tests but requires only what is necessary for determining a patient's health care needs. ${ }^{23}$

CMS provides no guidance as to what to do or not do in a preoperative assessment, but it does not reimburse for routine screening tests or for age-based testing. ${ }^{24}$ Reimbursement for a preoperative ECG, for example, requires documentation of the patient's signs or symptoms; for an ECG that is indicated, reimbursement includes review and interpretation by the physician. ${ }^{25}$

A new partner for proper preoperative assessment Appropriate preoperative evaluation and testing is one of the goals promoted by the recently formed Society for Perioperative Assessment and Quality Improvement (SPAQI). The mission of this international nonprofit organization is to optimize surgical outcomes by sharing best practices and promoting research and communication among health professionals across multiple disciplines. More information is available at www.spaqi.org. 


\section{DISCUSSION}

Question from the audience: At my hospital, we teach residents about limiting the preoperative tests they order, but surgeons routinely expect many of these tests, including chest $\mathrm{x}$-rays in patients with pulmonary conditions. Are any surgical societies involved in efforts to reduce preoperative testing? Or are surgical societies' recommendations actually driving some of the unnecessary testing?

Dr. Hepner: I'm not aware of recommendations from surgical societies regarding preoperative testing. Many surgeons believe that the more testing that's done, the likelier they are to uncover an occult disease. They also often want baseline information, which may actually be warranted in some cases.

Question from the audience: If you're already ordering a "type and screen" or "type and hold" for a patient, isn't it worthwhile to just add on a CBC? The patient is already getting the phlebotomy, so isn't there a cost benefit to getting other routine tests done at the same time rather than calling the patient back for more tests if another indication arises?

Dr. Hepner: Charges are generally assessed for each individual test, not for drawing blood, so I would only get the tests that are indicated.

Question from the audience: In institutions without a preoperative clinic, sometimes the surgeons do the workup without discussing the case with the primary doctor, and the surgeons want an ECG so that the case isn't cancelled at the last minute. Can you give straightforward criteria in such cases, such as an age threshold, or would you not order an ECG for anyone?

Dr. Hepner: Based on our most recent data, 60 years seems to be a reasonable cutoff if you are going to use age as a criterion.

Question from the audience: What criteria do you use for preoperative screening with pregnancy tests?

Dr. Hepner: If you have an unreliable patient population, general screening should be done. We don't have such a requirement, but we have a very low threshold. If a patient appears very reliable, knowing the exact date of her last menstrual period, we'll go by that. If a patient is unsure, we'll do a pregnancy test.

Question from the audience: My hospital doesn't have a preoperative clinic, and until recently, the anesthesiology department has helped surgeons with ordering of preoperative tests. We followed a guideline protocol for about 20 years. Now the newer surgeons say they don't want to be responsible for abnormal test results. Yet we anesthesiologists aren't seeing the patients, so we can't use clinical judgment; we can only go by the guidelines.
The surgeons are the only physicians on the case who actually do the history and physical exam. So who should sign the test orders and be responsible for abnormal results?

Dr. Hepner: In our preoperative test center, we tell the surgical team that if they are uncertain about which tests to order, we will handle it. And if we order a test, we follow up on the results. You must ensure that orders are signed and not rubber-stamped; that way, the person who orders a test will get called with any abnormal results. If you order it, you own it.

Question from the audience: I agree that no testing is needed for the 72-year-old man you presented who was undergoing surgery for inguinal hernia, but it always worries me not to do an ECG since part of the standard of care for anesthesia is intraoperative ECG monitoring. If we see some sort of unusual arrhythmia when we take the patient in, we might cancel the case if we don't know whether it was present at baseline. Surgeons will ask me, "Why didn't you order a baseline if you're going to monitor the ECG in the operating room? If you're not going to order a baseline, then why monitor the ECG?" These are medicolegal issues that I haven't seen addressed.

Dr. Hepner: A case like you describe will be addressed in the upcoming medicolegal session (see page S119). You make a good point that many times just having a baseline is helpful, but I would argue that it is more helpful for intermediate- or high-risk cases.

\section{DISCLOSURES}

Dr. Hepner has indicated that he has no financial relationships with commercial interests that have a direct bearing on the subject matter of this article.

This article was developed from an audio transcript of Dr. Hepner's lecture at the 4th Annual Perioperative Medicine Summit. The transcript was edited by the Cleveland Clinic Journal of Medicine staff for clarity and conciseness, and was then reviewed, revised, and approved by Dr. Hepner.

\section{REFERENCES}

1. Klafta JM, Roizen MF. Current understanding of patients' attitudes toward and preparation for anesthesia: a review. Anesth Analg 1996; 83:1314-1321.

2. Halaszynski TM, Juda R, Silverman DG. Optimizing postoperative outcomes with efficient preoperative assessment and management. Crit Care Med 2004; 32(suppl 4):S76-S86.

3. Roizen MF, Kaplan EB, Schreider BD, Lichtor LJ, Orkin FK. The relative roles of the history and physical examination, and laboratory testing in preoperative evaluation for outpatient surgery: the "Starling" curve of preoperative laboratory testing. Anesthesiol Clin North Am 1987; 5:15-34.

4. Roizen MF. More preoperative assessment by physicians and less by laboratory tests. N Engl J Med 2000; 342:204-205.

5. Schein OD, Katz J, Bass EB, et al. The value of routine preoperative medical testing before cataract surgery. Study of Medical Testing for Cataract Surgery. N Engl J Med 2000; 342:168-175.

6. Fischer SP. Development and effectiveness of an anesthesia preoperative evaluation clinic in a teaching hospital. Anesthesiology 1996; 85:196-206.

7. Finegan BA, Rashiq S, McAlister FA, O’Connor P. Selective 
ordering of preoperative investigations by anesthesiologists reduces the number and cost of tests. Can J Anaesth 2005; 52:575-580.

8. Dzankic S, Pastor D, Gonzalez C, Leung JM. The prevalence and predictive value of abnormal preoperative laboratory tests in elderly surgical patients. Anesth Analg 2001; 93:301-308.

9. Liu LL, Dzankic S, Leung JM. Preoperative electrocardiogram abnormalities do not predict postoperative cardiac complications in geriatric surgical patients. J Am Geriatr Soc 2002; 50:1186-1191.

10. American Society of Anesthesiologists Task Force on Preanesthesia Evaluation. Practice advisory for preanesthesia evaluation: a report by the American Society of Anesthesiologists Task Force on Preanesthesia Evaluation. Anesthesiology 2002; 96:485-496.

11. Silverstein MD, Boland BJ. Conceptual framework for evaluating laboratory tests: case-finding in ambulatory patients. Clin Chem 1994; 40:1621-1627.

12. van Klei WA, Bryson GL, Yang $\mathrm{H}$, et al. The value of routine preoperative electrocardiography in predicting myocardial infarction after noncardiac surgery. Ann Surg 2007; 246:165-170.

13. Correll DJ, Hepner DL, Chang C, Tsen L, Hevelone ND, Bader AM. Preoperative electrocardiograms: patient factors predictive of abnormalities. Anesthesiology 2009; 110:1217-1222.

14. Fleisher LA, Beckman JA, Brown KA, et al. ACC/AHA 2007 guidelines on perioperative cardiovascular evaluation and care for noncardiac surgery: executive summary. J Am Coll Cardiol 2007; 50:1707-1732.

15. U.S. Preventive Services Task Force. Screening for coronary heart disease: recommendation statement. Agency for Healthcare Research and Quality Web site. http://www.ahrq.gov/clinic/3rduspstf/chd/ chdrs.htm. Accessed March 20, 2009.

16. Hepner DL, Bader AR, Correll D, Tsen LC, Segal BS, Bader AM. An analysis of preoperative testing protocols in academic anesthesiology programs. Poster presented at: 4th Annual Perioperative Medicine Summit; February 5-7, 2009; Miami Beach, FL. http://
www.ccjm.org/content/76/Electronic_Suppl_1/eS18.full.pdf+html. Accessed May 28, 2009

17. De Hert SG. Preoperative electrocardiograms: obsolete or still useful? Anesthesiology 2009; 110:1205-1206.

18. Fleisher LA. The preoperative electrocardiogram: what is the role in 2007? Ann Surg 2007; 246:171-172.

19. Rich MW. The preoperative electrocardiogram: have we reached the end of an era? J Am Geriatr Soc 2002; 50:1301-1303.

20. Noordzij PG, Boersma E, Bax JJ, et al. Prognostic value of routine preoperative electrocardiography in patients undergoing noncardiac surgery. Am J Cardiol 2006; 97:1103-1106.

21. Ferschl MB, Tung A, Sweitzer B, Huo D, Glick DB. Preoperative clinic visits reduce operating room cancellations and delays. Anesthesiology 2005; 103:855-859.

22. Correll DJ, Bader AM, Hull MW, Tsen LC, Hepner DL. The value of preoperative clinic visits in identifying issues with potential impact on operating room efficiency. Anesthesiology 2006; 105:1254-1259.

23. The Joint Commission. 2009 Comprehensive Accreditation Manual for Hospitals: The Official Handbook. Oak Brook, IL: Joint Commission Resources; 2008.

24. Medicare coverage center. Centers for Medicare \& Medicaid Services Web site. http://www.cms.hhs.gov/Center/coverage.asp. Accessed March 20, 2009.

25. CMS Manual System: Pub. 100-03 Medicare National Coverage Determinations. Dept of Health \& Human Services (DHHS), Centers for Medicare \& Medicaid Services (CMS). Transmittal 26. December 10, 2004.

Correspondence: David L. Hepner, MD, Brigham and Women's Hospital, Department of Anesthesiology, Perioperative and Pain Medicine, 75 Francis Street, CWN L1, Boston, MA 02115; dhepner@partners.org 